Rapport - Société canadienne d'histoire de l'Église catholique

\title{
Le docteur Joseph-Raoul Hurtubise, M.D., Sénateur
}

\section{Guy Courteau}

Volume 27, 1960

URI : https://id.erudit.org/iderudit/1007403ar

DOI : https://doi.org/10.7202/1007403ar

Aller au sommaire du numéro

Éditeur(s)

La Société canadienne d'histoire de l'Église catholique

ISSN

0318-6148 (imprimé)

1927-7075 (numérique)

Découvrir la revue

Citer cet article

Courteau, G. (1960). Le docteur Joseph-Raoul Hurtubise, M.D., Sénateur. Rapport - Société canadienne d'histoire de l'Église catholique, 27, 53-70.

https://doi.org/10.7202/1007403ar

Tous droits réservés @ La Société canadienne d'histoire de l'Église catholique, 1961
Ce document est protégé par la loi sur le droit d'auteur. L'utilisation des services d'Érudit (y compris la reproduction) est assujettie à sa politique d'utilisation que vous pouvez consulter en ligne.

https://apropos.erudit.org/fr/usagers/politique-dutilisation/ 


\section{Le docteur Joseph-Raoul Hurtubise, M.D., Sénateur}

\section{SOURCES}

1. Manuscrites :

Archives du Collège du Sacré-Cœur, citées sous le sigle ACSC.

Le Fonds Hurtubise, indiqué par le sigle FH, comprend l'autobiographie du docteur Raoul Hurtubise, sa généalogie, sa correspondance, ses discours à North Bay, 26 nov. 1944; à l'hôpital Saint-Joseph de Sudbury, 1946; à Windsor, lors du dévoilement du monument à Jeanne Mance, 27 oct. 1946; Spicilc̀ges (albums à découpures) contiennent de nombreuses références aux périodiques suivants : le Droit, 2 fév. 1955; la Feuille d'Erable, fév. 1955; la Frontière, 21 juin 1945; le Lien, mars 1955; The Sudbury Daily Star, 25 juillet 1945.

\section{IMPRIMÉES :}

Un demi-siècle d'activité (1896-1946) à l'hôpital Saint-Joseph, Sudbury, 1946.

Souvenir du Cinquantenaire (1883-1933) de la paroisse Sainte-Anne, Sudbury, 1933.

Cadieux, Lorenzo, s.j., Fondateurs du diocèse du Sault-Sainte-Marie, Sudbury, La Société historique du Nouvel-Ontario, 1944, Coll. Documents historiques, $\mathbf{n}^{\circ} \mathbf{6}$; idem, Missionnaires au lac Nipigon, 1957, Coll. Documents historiques, $\mathrm{n}^{\circ} 33$.

Hurtubise, J.-Raoul, Le $D^{r}$ William $H$. Howey, voir Familles pionnières, Sudbury, La Société historique, 1944, Coll. Documents historiques, $\mathrm{n}^{\circ}$ 5; idem, Ecoles bilingues de Sudbury, Sudbury, La Société historique, 1954, Coll. Documents historiques, $\mathrm{n}^{\circ} 28$ : 21-42.

Lamarche, Gustave, c.s.v., Le Collège sur la Colline, Petit historique du Collège Bourget, Rigaud, Ed. de l'Echo de Bourget, 1951.

Normandin, Pierre-Georges, The Canadian Parliamentary Guide, Ottawa, 1954: 83-84.

Raymond, Alphonse, s.j., Paroisse Sainte-Anne, Sudbury, La Société historique, 1953, Coll. Documents historiques, $\mathrm{n}^{\circ} 26$.

Au cours de congrès antérieurs, le R.P. Lorenzo Cadieux, s.j., le président général de votre méritante Société, a déjà synthétisé l'œuvre des Jésuites missionnaires dans ce diocèse. Relisez ses deux remarquables travaux : Fondateurs $d u$ diocèse $d u$ Sault-Sainte-Marie et Missionnaires au lac Nipigon ${ }^{1}$.

D'autres, en ce présent congrès, esquisseront l'œuvre de leurs zélés continuateurs, les membres du clergé séculier.

1 Lorenzo Cadieux, s.j., Fondateurs du diocèse du Sault-Sainte-Marie, Sudbury, La Société historique du Nouvel-Ontario, 1944, Coll. Documents historiques, $\mathrm{n}^{\circ}$ 6; idem, Missionnaires au lac Nipigon, 1957, Coll. Documents historiques, $\mathbf{n}^{\circ} 33$. 
D'aucuns se demanderont peut-être pourquoi le Sénateur Hurtubise figure-t.il au programme? Je répondrai : pour plusieurs raisons. D'abord, parce qu'il était presque un homme d'Eglise. Puis pour d'autres raisons que j'étalerai le long de cet essai de biographie. Pour le moment, permettez-moi d'en souligner une très spéciale qui intéresse au plus haut point votre Société. N'en fut-il pas tour à tour et à plusieurs reprises directeur, vice-président et président?

Il fut aussi membre fondateur et premier président de la Société

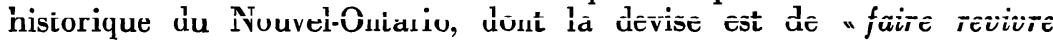
notre histoire ".

Vous présenter le docteur Hurtubise, c'est donc évoquer une tranche de notre histoire locale, à la fois civile et religieuse; c'est par le fait même retracer un demi-siècle de l'histoire du Nouvel-Ontario, dont il fut, sans contredit, une figure de proue. Il rivalisait de zèle apostolique avec les meilleurs prêtres patriotes de son temps - ses grands amis - dans la défense de la cause française et catholique. Et cette Eglise catholique dont votre Société se fait un devoir de ressusciter l'histoire, n'est-elle pas composée de prêtres et de laïcs ?

Les différentes sources consultées, tant manuscrites qu'orales, m'ont parlé de lui en termes élogieux. Toutes l'ont acclamé d'une voix unanime l'un des chefs les plus sympathiques de la minorité francoontarienne. Toutes m'ont fourni l'ultime explication du secret de cette vie, qui m'a paru parfois comme une sorte de défi ou de gageure : vigueur suave, humilité et charité, à dose peu commune, en étoffaient la contexture.

Comme le jour est en germe dans l'aurore, ainsi la vie publique ordinairement s'éclaire à fond par la vie privée. Cette vie intime nous renseignera sur l'enfance et la formation du docteur Hurtubise. Elle nous révélera le milieu précis où évolueront le professionnel et le catholique exemplaire.

\section{VIE PRIVÉE (1883-1930) ${ }^{2}$}

Joseph-Raoul Hurtubise naquit le $1^{\text {er }}$ juillet 1882, à Sainte-Annede-Prescott (Ontario). Il était le sixième enfant d'une famille de treize, dont huit garçons et cinq filles. Les deux premiers-nés étant morts en bas âge, il acquit vite le privilège du droit d'aînesse. Un de ses frères, Henri, devint prêtre, un autre médecin, puis une religieuse, sœur SaintConstant, chez les Sours Grises de la Croix d'Ottawa; les autres, mariés pour la plupart, restèrent dans le monde.

Son père, né à Saint-Rédempteur, s'était marié à 35 ans, le 11 mai 1874. Après avoir travaillé dans les chantiers, il acquérait une terre de bois franc, de formation rocailleuse, à Sainte-Anne-de-Prescott. Il la défrichait et l'ensemençait l'été, puis retournait aux chantiers l'hiver. Là, il passait pour un maître équarrisseur. Pendant quarante

2 ACSC, FH, A : 1-10. 
ans, il peina pour « joindre les deux bouts ». Grand chrétien, il était d'un caractère \& calme, patient, industrieux, courageux, tenace, toujours confiant dans l'avenir ». Il mourut à 69 ans.

Sa mère, Casildé Lemaire dit Rapidiou, épousait Joseph Hurtubise à 21 ans et le suivit pas à pas. Les travaux variés de la ferme, joints au jardinage et à l'élevage des animaux (vaches, veaux, moutons et porcs) comme des volailles et des dindons, alternaient avec les multiples besognes domestiques comprenant, en plus du soin de la progéniture, la cuisson des pâtes et du pain au four, le barattage du beurre, le tissage au métier, le filage au rouet, la broderie au crochet sans compter les sempiternels lavages et raccommodages. Elle s'éteignit le 14 janvier 1908, à l'âge de 45 ans, mais vécus en double. Elle avait toutefois réalisé sa noble ambition " d'élever de bons enfants et leur donner toute l'instruction possible».

De tels parents marquent une âme d'enfant pour la vie. Comme le chêne vit de la qualité de la terre où il s'enracine, Raoul vivra du souvenir de l'ineffable vertu de ces âmes de paysans qui ont saintement façonné la sienne...

Ses parents, riches en trésors spirituels, mais pauvres en biens de la terre, avaient à cœur cependant de pourvoir équitablement à la subsistance comme à l'éducation de leurs mioches.

La montée vers la vie (1883-1908)

Malgré une santé débile et dès l'âge de six ans à peine, Raoul, talent précoce, montait vers la vie et fréquentait l'école du rang, à un mille et demi de la maison paternelle. Pour se familiariser avec l'anglais, il suivit, durant les années 1895-1897, des cours en cette langue à Alexandria. Pendant ce séjour, il servit la messe de M. l'abbé Elie Poitras, premier prêtre canadien-français de la cathédrale qui desservait ses compatriotes. Ce dernier multiplia les instances auprès de son servant pour le faire entrer au Juniorat des RR.PP. Oblats, à Ottawa. Au printemps de 1897, ses parents décidèrent plutôt de lui faire poursuivre des études classiques. Comme préparation, le curé de la paroisse, M. l'abbé Joseph Coderre, "un homme charitable au possible », lui enseigna des leçons de syntaxe ${ }^{3}$.

L'automne suivant, il était admis aux Eléments-Latins dans le "Collège sur la Colline" ou Bourget, à Rigaud, Québec, pour la modique somme de cent dollars par an, études et pension comprises. Dieu aidant, et avec beaucoup de persévérance, - car sa santé laissait toujours à désirer, - il put parfaire ses études et, en 1904, obtenir son baccalauréat ès Arts, de Laval, avec grande distinction.

Au collège, la Providence l'entoura d'une élite d'éducateurs zélés 4 . Tous, moins un, étaient des Clercs de Saint-Viateur.

3 Lettre de Gustave Lamarche, c.s.v., à Guy Courteau, s.j., 12 septembre 1960.

4 G. Lamarche, Le Collège sur la Colline, passim. 
Bien qu'il fût élevé sous le second régime Charlebois, de 1897 à 1901, un géant de l'action à la fois poète et musicien, puis futur Provincial de Chicago et enfin de Montréal et qu'il dût profiter beaucoup au contact de cet artiste, il semble bien que c'est le R.P. Alphonse-Donat Richard, de 1901 à 1904, qui l'a influencé le plus. Confident du père Charlebois, et son bras droit, il occupe dans l'histoire du Collège une des tranches imposantes, qu'on a justement appelée : l'époque Richard (1901-1916).

Grand seigneur et natriote, ret homme fera toujourc l'admiration du jeune Hurtubise. Plus tard, il conservera de constantes relations avec lui. Dans la lutte contre le Règlement XVII, le R.P. Richard demeurera l'une des figures dominantes du clergé québecois.

Dès le Collège, ceux que la Providence destine à jouer un rôle primordial se préparent sérieusement et manifestent déjà des traits de leur personnalité naissante. "C'est à la dernière "séance " du régime Charlebois que Raoul Hurtubise allait commencer son ascension. Il s'habillait en effet, ce jour-là, 20 mars 1901, en Chevalier du Mont SaintMichel, dans la Revanche de Jeanne d'Arc. Trois ans plus tard, c'est lui qui présenterait la maison Richard au père Lajoie, supérieur général, en un "fort beau compliment ", dit le journal du père Richard ${ }^{5}$. " Ceci montre de quelle estime on l'auréolait...

Quand le jeune homme monte vers la maturité, surgit la question capitale du choix d'une carrière. Il opta pour la médecine, à la grande déception de sa mère, qui avait espéré ardemment la prêtrise...

L'été suivant, Raoul gagna Piercefield (New York) et s'engagea dans un moulin à pulpe, afin d'amasser quelque pécule et aider ainsi ses parents à défrayer en partie le coût de l'Université.

Malgré cette aide, le père, chef d'une famille nombreuse, se trouvait dans l'impossibilité de lui payer des études médicales. Ne possédant qu'une terre de cent arpents, il avait à solder en outre les frais d'instruction des cadets, Elzéar et Henri, déjà inscrits au Collège. Le père décida de léguer sa ferme à Joseph, le troisième des garçons qui avait toujours cultivé avec son père, à condition que le fermier se chargeât de payer à ses autres frères Université et Collège. Néanmoins, afin que tous ses enfants reçoivent une part juste et équitable au point de vue instruction, Raoul qui, pour le moment, recevait davantage, devait s'engager en retour, dès que son cours universitaire serait complété et qu'il serait en mesure de le faire, à donner la somme de deux cents dollars à chacune de ses søurs.

La Providence aidant, et grâce à Joseph-Raoul, à l'automne de 1904, s'inscrivait en médecine à l'Université Queen's de Kingston (Ontario). Son séjour y fut très agréable. Les autorités, des presbytériens écossais (Scotch Presbyterians), ne faisaient aucune distinction de nationalité ou de religion... Les étudiants étaient traités sur un pied

5 Ibid. : 109, 141. 
d'égalité. Les Canadiens français y étaient clairsemés, dont quatre en première année : MM. Edmond Charbonneau, Albert Charlebois, P.-O. Coulombe et J.-R. Hurtubise.

Comme bon nombre d'étudiants, Raoul comptait ses sous. Il subit parfois des jours sombres et rien ne lui était plus pénible que d'avoir à quémander des subsides à ses parents. Il devinait d'avance tous les sacrifices que ces laborieux s'imposeraient pour satisfaire à sa requête et boucler quand même le budget... Un jour même, afin de pouvoir achever ses études universitaires, sans trop mettre à la gêne ces derniers, il dut recourir aux bons offices d'un voisin, M. Noé Deschamps, et lui emprunter l'appoint.

Au printemps de 1908, il reçut son doctorat en médecine et en chirurgie. Le jour de la collation des diplômes, le doyen de l'Université Queen's, M. G. C. Connell, le fit mander et lui proposa un emploi, à titre de médecin, à la mine Wilbur Iron Ore Company, à Wilbur, Ontario. Les honoraires étaient de cent dollars par mois, toutes dépenses payées. Deux jours plus tard, il entrait en fonction. Il fut installé en pleine forêt, au milieu d'une population cosmopolite. Ses chefs étaient gentils, bienveillants et serviables.

Au cours des deux premiers mois, et dans l'intention d'économiser, le jeune médecin n'endossa pas ses chèques de paye, attendant la première occasion de se rendre au village le plus proche y déposer le montant à la banque. Malheureusement, le troisième mois, la compagnie fit faillite et, comme il y avait très peu d'argent en caisse, elle paya les journaliers les premiers. Quand vint le tour du médecin, la caisse était vide et le jeune Hurtubise perdit le fruit de ses labeurs. Il dut emprunter pour retourner chez lui...

Durant les années de collège et d'université, il passait ses vacances à travailler à la ferme : réparation des bâtiments, trayage des vaches, peinturage, etc. Comme aîné, il détenait l'onéreux privilège d'être le dernier dans la répartition des tâches.

L'année 1908 fut particulièrement mémorable, mais pénible. Le 14 janvier, après un assez long martyre, mourait sa mère dans la quarantaine et, le 24 novembre, c'était le tour de son père. Au printemps, comme nous venons de le voir, Raoul avait bien reçu son doctorat en médecine, mais après la désillusion de Wilbur, il revient chez lui désemparé, démonté...

"Mais ici encore, la Providence, cette chère et divine Providence » vint à son secours. Une connaissance, le docteur Joseph Bédard, M.D., de North Bay, lui conseilla de s'établir à Verner. Il boucle immédiatement ses malles, quitte Sainte-Anne-de-Prescott et file vers cette nouvelle étoile qui brillait comme une étincelle d'espoir dans sa nuit... Un obstacle l'attendait : le docteur Dafoe y était installé. Le docteur Bédard, jamais à court d'expédients, proposa au docteur Dafoe, un anglo-protestant, un marché plus alléchant que Verner, paroisse totalement catholique et française... Dafoe accepta et laissa le champ libre 
au docteur Hurtubise... Décidément, la Providence - cette divine et très chère Providence, comme il l'appelle, - s'en mêlait...

Avec l'automne de 1908, débute une nouvelle tranche de l'histoire de notre héros : l'entrée en scène du professionnel dans le NouvelOntario, milieu jeune et prometteur.

Ecoutons le docteur nous le décrire avec sa précision coutumière. Il nous avertit de ne pas confondre "l'Ontario-Nord ou l'extrême nord avec le Nouvel-Ontario ou le "sud du nord ", dont voici les délimitations géographiques. Le Nouvel-Ontario s'étend de la rivière Ottawa à Mattawa, jusqu'à la baie Georgienne et au lac Supérieur, tout le long des voies de chemin de fer. Il est limité au sud par le lac Nipissing et la rivière des Français, au nord par la hauteur des terres ${ }^{6}$."

Dans la description des localités, il ne manque pas de caractériser Verner. Le docteur Hurtubise y résidera deux ans, de décembre 1908 à janvier 1910. Il visita les malades de la place et des environs en voiture, "bogie ou cutter", par des chemins de terre. Au milieu de cette vie plutôt rude, le bon accueil, la franchise et la simplicité de ses patients le consolaient. Au mois de janvier 1910, à la supplique de sœur Marie-Ephrem, supérieure de l'hôpital Saint-Joseph de Sudbury, le docteur de campagne transporta ses pénates dans cette petite ville naissante... Il quitta Verner avec regret conservant de tous le plus doux souvenir.

\section{A Sudbury...}

Il ouvrit un bureau au premier étage de l'édifice Frawley. Il lui fallut débuter de nouveau : se refaire une clientèle et, en même temps, prodiguer ses soins aux patients de l'hôpital. La ville de Sudbury comptait alors une population de 4,000 âmes; les rues n'étaient pas macadamisées et les trottoirs étaient en bois. Il y avait passablement de boue et de poussière. La nuit, il devait répondre aux appels venant de Hanmer, Blezard, Rayside, Chelmsford, Morgan, Lac Vermillon, Azilda, Larch Wood, Broder, Coniston, Garson, etc... Il faisait ses trajets en voiture ou se rendait par train à Cartier, Markstay, Sellwood, etc... De plus, en hiver, durant les deux premières années, il fit même la visite des chantiers. Ici, rendons hommage au médecin de campagne, le digne émule du docteur W. Howey, premier médecin de Sudbury. Les traits qu'il lui a attribués se confondent avec les siens. N'a-t-il pas écrit : "Le docteur Howey était bon jusqu'à l'oubli de soi-même" et "il était l'ami de tout le monde et l'ennemi de personne ».

\section{A l'hôpital...}

Dirigé par les RR.SS. Grises de la Croix d'Ottawa, l'hôpital SaintJoseph ne possédait que quatre-vingts lits et ne comptait en fait de gardemalades diplômées que celles qui faisaient du service privé. Ses premiers

- Raoul Hurtubise, Les Canadiens français et le Nouvel-Ontario, Montréal, Coll. L'Euvre des Tracts, $\mathrm{n}^{\circ} 244$ (oct. 1939) : 1-6. 
confrères furent les docteurs W. H. Mulligan, D. R. Paterson, et le docteur H. M. Torrington; puis à l'hôpital du Pacifique Canadien, les docteurs R. H. Arthur et W. J. Cook, et enfin McCauley et W. C. Morrison, de l'Inco, à Copper Cliff. Le docteur Howey pratiquait encore, mais s'abstenait de visites aux patients des hôpitaux.

Le docteur Hurtubise se mit à l'œuvre et débuta en chirurgie mineure. Il était très embarrassé par le manque d'installations matérielles modernes. Il ne tendait qu'à un seul but : travailler ferme et améliorer la situation. Comme la bonne entente régnait parmi les confrères, ils réussirent à mettre leurs projets à exécution. On proposa aux RR.SS. Grises l'ouverture immédiate d'une école de formation des gardes-malades. Les autorités de la maison mère, malgré le manque d'espace, d'argent et de personnel enseignant, s'empressèrent d'exaucer ces desiderata. Au mois de septembre 1911, trois candidates s'inscrivirent; la future sœur Saint-Firmin, et les demoiselles Cécilia Cull et Ernestine Beauchênes. On nomma comme directrice la révérende sœur Eugénie, garde-malade diplômée. Les docteurs W. H. Mulligan, D. R. Paterson, H. M. Torrington et J.-R. Hurtubise se partagèrent les cours. Le docteur W. H. Mulligan devient le premier président du corps médical et le docteur Hurtubise, le secrétaire. En 1915 et en 1916, c'est lui qui remplacera le docteur Mulligan, il demeurera membre du personnel jusqu'en 1930.

Ce fut alors l'ouverture d'une ère de progrès. Le Pacifique Canadien ferma son hôpital, et ses médecins, Arthur et Cook, joignirent le personnel de l'hôpital Saint-Joseph.

C'est à ce moment, en 1917, que le docteur Hurtubise se prépara par de fortes études spéciales à la chirurgie générale ou majeure. Il passa trois mois au "New York Postgraduate Hospital ». En 1924, il fit un stage de deux mois chez les frères Mayo à Rochester; puis en 1925, dans les cliniques de Londres et de Paris, en compagnie des meilleurs maîtres. En 1926, après avoir présenté les travaux nécessaires devant l'aréopage des examinateurs, il obtient le titre envié de membre du Collège des Chirurgiens américains (Fellow of the American College of Surgeons-F.A.C.A.). Sans jamais abandonner pour autant la pratique de la médecine générale, il fit graduellement son ascension en chirurgie et s'y spécialisa.

\section{Portrait}

La prestance et la stature du professionnel contrastaient fort, sans doute, avec la santé débile de l'éphèbe et reflétait la robustesse de constitution. Une garde-malade l'a campé en raccourci par ces mots du cœur : "Il était un bon ami et un très beau gentilhomme... " Elle n'était pas la seule à penser ainsi.

Dans les soirées, quel naturel jovial, affable, conciliant et pacifique !

Partout, quelle âme transparente d'enfant dont la candeur se refuse à toute pactisation avec l'ombre même de la méchanceté ! Le brillant causeur savait écouter les autres et les encourager. Il avait l'art 
d'admirer, un tempérament d'artiste avec la figure et l'âme d'un grand seigneur d'autrefois, "mentonnette » impériale comprise! De sa voix de basse, il aimait à chanter à la mode d'autrefois, quand la chanson et les jeux de société avec les charmes de la conversation soignée tenaient lieu de cinéma, radio et T.V. Une de ses chansons préférées c'était, dit-on, " Le nid d'amour »...

Au sein d'une famille nombreuse il avait appris de bonne heure, avec l'esprit de travail et de sacrifice, le sens du devoir et du dévouement, fines fleurs du sons sncial Ohligeant nour tous. il sera toute sa vie d'une politesse exquise où dominait un parfait mélange de douceur et de bonté, qu'il avait hérité de ses parents et peut-être aussi de la révérende mère Saint-Raphaël, la troisième supérieure de l'hôpital SaintJoseph qui l'avait accueilli à son arrivée.

Généreux jusqu'à la libéralité, il prodiguera gratuitement ses soins aux indigents, exposera ses jours en passant des nuits entières au chevet des malades, lors de l'épidémie de grippe espagnole ou influenza, en 1918-1919. Il soutenait aussi les membres de sa famille et paya largement pour l'instruction de plusieurs de ses neveux et nièces.

A Sainte-Anne, certaines jeunes filles, moins fortunées, qui voulaient entrer en religion, savaient où frapper pour compléter la dot requise, etc.

Si l'on ajoute à ces nombreuses qualités, une humilité et une intégrité qui ne se démentiront jamais, le portrait sera ressemblant.

Je n'ai fait qu'ébaucher ici les traits saillants de sa physionomie, quitte à les retoucher en filigrane à mesure qu'aux prises avec les événements et la maturité, ces traits s'accuseront plus nettement.

\section{Le professionnel}

Comme médecin praticien et consultant, il gagnait la confiance et l'amitié aussi bien de ses confrères que de ses patients par la compétence et la sereine bonhomie inoubliable de son accueil. On percevait vite qu'il concevait la médecine «comme une sorte de sacerdoce. Cette haute conception inspirait toute sa conduite ${ }^{7}$."

Clinicien ponctuel et supérieurement doué, il avait acquis une solide expérience auprès des maîtres les plus réputés d'Europe, des Etats-Unis et du Canada. Il pratiqua régulièrement, même l'anesthésie. Psychologue, il faisait compter fort le malade avec lui, puis ralentissait et abaissait sa voix à mesure que l'éther agissait, afin de ne pas trop incommoder ou suffoquer le patient. Entre temps, de 1910 à 1930, il communiquait ses vastes connaissances, car il était en outre professeur d'anatomie chez les garde-malades.

Ce chirurgien consciencieux, qui maniait le scalpel avec autant de sûreté que d'habileté. n'en suivait pas moins régulièrement ses malades sans distinction de classes. Ses opérés, il les aimait paternellement et

7 Camille L'Heuneux, Un chef franco-ontarien disparaît, dans le Droit, 2 février 1915. 
leur faisait tant de bien que souvent il procura double guérison : la santé de l'âme est si étroitement liée à celle du corps. Grand croyant, il pouvait dire après Ambroise Paré : « Je le pansay, Dieu le guarist...» Il devint si habile que ses confrères les plus jeunes, les docteurs Lévesque, Tanguay et autres, parlaient couramment de la " méthode Hurtubise " et dans des cas difficiles, abandonnaient même des méthodes parisiennes et revenaient à cette méthode comme à la meilleure... ${ }^{8}$

En 1946, les directeurs de l'Association des Hôpitaux de l'Ontario s'honoraient en l'élisant membre de leur bureau ${ }^{9}$.

\section{Le catholique exemplaire}

Ce maître-chirurgien, d'une vaste culture humaniste, était un croyant convaincu, un pratiquant sincère. Sa foi vive s'appuie sur l'abandon filial à la sainte Providence. Ce mot revient souvent dans ses écrits et il l'emploie en termes émus qui ne trompent pas... Même à travers les plus rudes épreuves, il n'a pas douté un seul instant de son Dieu, du Père qui veille avec amour sur tous ses enfants.

Il centrait sa vie sur la messe et la communion et ne manquait jamais d'assister à la grand-messe, le dimanche et souvent à la basse sur semaine, quand ses occupations lui en laissaient le loisir.

Le docteur employait tous ses moments libres à promouvoir la vie intense de la paroisse. Il était de tous les mouvements et contribuait largement de sa personne et de sa bourse, mais sans ostentation. Il intervint dans la question de la division de la paroisse, de 1915 à 1919.

Le 18 septembre 1934, le député de Nipissing se faisant l'écho et le défenseur de ses compatriotes, écrit au Délégué apostolique une longue lettre documentée sur la situation des Canadiens français du diocèse et suggère un remède radical. Les événements lui ont donné raison. Lors de la visite du même délégué apostolique, $\mathrm{M}^{\mathrm{gr}}$ Andréa Cassulo, un an auparavant, c'est lui qui avait été choisi pour lire l'adresse de bienvenue au nom des paroissiens.

Paroissien modèle, il vénérait tous les membres du clergé en général ${ }^{10}$ et les Jésuites en particulier. Il recherchait leurs directives en tout et les suivait fidèlement. De petits faits ont parfois une immense portée. Dans ses notes, il avait dressé la liste des curés et des vicaires de Sainte-Anne de Sudbury et c'est lui qui donna, bien encadrée, la galerie des portraits de tous les curés pour ornementer les murs de la salle paroissiale ${ }^{11}$.

En maintes occasions, il a montré son admiration et son estime pour la Compagnie de Jésus. Sa mère avait vu juste : Raoul avait une

8 Julien Morisetre, Le sénateur Hurtubise, dans la Frontière, 21 juin 1945.

The Sudbury Daily Star, 24 octobre 1945.

10 Hurtubise, op. cit. : 15s.

11 A. Raymond, s.j., Paroisse Sainte-Anne de Sudbury, 1953, Coll. Documents historiques, $\mathrm{n}^{\circ} 26$. 
âme de prêtre. Son frère Henri n'avait pas tort non plus de renchérir en l'appelant « Docteur Ignace ${ }^{12}$ ».

Badinage à part, rien de plus apparentée d'ailleurs que l'âme du médecin et du prêtre. Ainsi le docteur Schweitzer sympathisait d'emblée avec le missionnaire. Tous les deux n'ont-ils pas les mêmes motifs de vivre : se vouer sans merci au soulagement de la misère humaine ?...

S'il est de tous les mouvements corvéables, il est aussi à l'honneur dans toutes les fêtes. C'est qu'il a aimé sa paroisse adoptive comme une seconde patrie. Aux cinquantièmes anniversaires, soit de l'hôpital SaintJoseph en 1946, soit de la paroisse Sainte-Anne en 1933, soit lors du centenaire de la Compagnie de Jésus en 1941, il est au nombre des orateurs ou joue un rôle prépondérant. Il exalte sans réserve l'œuvre des Jésuites, missionnaires et éducateurs, puis l'œuvre des RR.SS. Grises. On sent vibrer son âme.

Magnanime, ce professionnel avait un sens chrétien profond et pratiquait le grand commandement de la charité. Personne à Sudbury n'a mieux compris et incarné ce mot du Christ : "Apprenez de moi, car je suis doux et humble de cœur. " Il avait le regard aimant des humbles et le promenait affectueusement sur tout son entourage.

Les curés de Sainte-Anne et les recteurs du Collège Sacré-Cœur comme les RR.SS. Grises n'ont cessé de reconnaître hautement la forte personnalité de ce grand chrétien.

\section{VIE PUBLIQUE (1930-1955)}

Les fonctions, comme les événements, ne forgent pas un homme, mais révèlent sur un plus vaste théâtre sa réelle valeur. trop fort?

Examinons le sacrifié dans le monde politique. Le mot est-il

Chose certaine, il n'est pas entré dans ce nouveau monde pour satisfaire ses goûts et ses ambitions ${ }^{13}$. Car de toute évidence, personnellement, il préférait au monde politique le monde médical dont il était le type achevé.

Là, comme ailleurs, il a, opinons-nous, obéi à une volonté impérieuse, à des motifs supérieurs. Il s'est oublié lui-même pour mieux servir les siens, croyant qu'un champ plus vaste s'ouvrirait ainsi à la défense de la cause catholique et française.

En apparence, il s'est rendu aux fortes pressions réitérées de ses amis les plus chers; puis, en dernier ressort, aux vœux des stratèges libéraux du temps.

Parmi ses amis, pour ne mentionner que les principaux, il y eut la voix persuasive et impérative du R.P. Charles Charlebois, o.m.i., et du

12 Lettre de H. Hurtubise à R. Hurtubise, 10 mai 1916, ACSC, FH.

13 L'Heureux, ibid.; ACSC, FH, A : 11. 
sénateur Belcourt ${ }^{14}$. Sa première tentative fut malheureuse, en ce sens qu'il fut battu par Charles McCrea, ministre des Mines. Cependant, il avait haussé le débat, puisqu'il avait orienté la lutte contre le Règlement XVII.

Un peu plus tard, les stratèges libéraux, Lapointe et consorts, flairant la défaite de 1930, revinrent à la charge. Les mêmes motifs, invoqués plus haut, le décidèrent d'accepter d'entrer de nouveau en lice. Pour éviter que la défaite libérale tourne au désastre, il fallait s'assurer quelques fiefs en présentant des candidats sûrs, dont la personnalité irréprochable gagnerait même les adversaires (des " front men »). E.-A. Lapierre avait eu l'incontestable mérite d'avoir conquis sur l'adversaire tout le comté. Craignait-on dans les hautes sphères libérales qu'en ces jours périlleux, Lapierre ne pourrait plus le maintenir au parti, malgré toute la popularité dont il jouissait? Je l'ignore. Toujours est-il qu'on jugea plus avisé de présenter le docteur Hurtuhise. Lapierre se sacrifia et laissa le champ libre. Hurtubise fut élu, mais le parti libéral écrasé.

Le nouveau député de Nipissing battit son adversaire conservateur, Harry Morel, de Mattawa, par une majorité de 2,151 voix; et, en 1935, c'était encore la victoire et, cette fois, par 12,123 voix, une majorité accrue contre MM. MacNamara, conservateur, Jos. Levert, C.C.F., Carl Stewart, reconstructionniste, et Hill, communiste. En 1940, il triomphait de M. O. Larocque, conservateur, par 16,000 voix de majorité. Une vie nouvelle, plus fébrile, s'ouvrait devant lui, la vie du député, sinon du politicien. De ce dernier, il n'a jamais eu le tempérament.

Le docteur Hurtubise apportait du neuf dans le monde politique. Les médecins de renom étaient plutôt clairsemés autrefois sur le parquet de la Chambre des Communes. La politique semblait le fief des avocats. Aujourd'hui, elle élargit ses cadres et sent le besoin de compter des représentants de tous les corps de la société. Plus que tous les autres, les médecins, à cause de leur connaissance du cœur humain et des problèmes concernant la santé et l'hygiène, pourraient être un apport précieux dans beaucoup de domaines, comme le faisait judicieusement remarquer le docteur $R$. Tanguay ${ }^{15}$.

Toujours il se montra l'homme de tout le monde, excellent conseiller, pacificateur hors pair, homme de tact. Il a peut-être plus fait pour la cause des siens que certains apôtres qui, sous couleur de paix, travaillent bien plus pour des fleurs et des couronnes et des promesses de bonne entente que de réelles garanties de nos droits et de notre fierté. Il n'a jamais confondu le noble concept de la paix avec toute cette hommerie-là !...

C'est vrai qu'il s'était présenté à Ottawa ayant déjà une assez belle expérience de l'administration de la chose publique. Il avait «un esprit rompu à la résistance et à la solution des problèmes complexes ${ }^{16}$. 》

14 Charles Charlebois, o.m.i., à Hurtubise, 14 février 1923; N.-A. Belcourt à Hurtubise, 24 avril 1923; R. Hurtubise à Belcourt, 30 avril 1923, ACSC, FH.

15 Eminent Surgeon, Politician Honored On Senate Elevation, dans The Sudbury Daily Star, 25 juillet 1945.

16 Morissette, ibid. 
Rappelons en bref quelques-unes de ses activités politiques. En 1931, il assista à l'ouverture officielle de l'extension - entre Cochrane et la Baie James - de la voie ferrée du T.N.O. (Témiscaming et Northern Ontario). En 1938, il représentait le gouvernement canadien avec Fred Sanderson au $150^{\circ}$ anniversaire de la Nouvelle-Galles du Sud, en Australie.

En 1939, lors de la réception officielle du roi et de la reine d'Angleterre à Sudbury, il lut une adresse à Leurs Majestés George VI et Eilizabeth.

Le 23 février 1943, le gouvernement le nomma dans le Comité parlementaire spécial des Assurances sociales d'après-guerre.

A partir de 1942, je cite Julien Morissette, "le député de Nipissing prit aux Communes certaines attitudes qui parurent étranges à ses commettants. C'est vrai qu'il représentait un comté aux allégeances mêlées... Avec la sincérité et la profonde honnêteté que je lui connais, j'ai la conviction qu'il les croyait les plus acceptables aux Canadiens français, même si à ce sujet, il a pu se tromper... Les jeunes se sont montrés cruels à Sudbury lui reprochant d'être un " suiveux ", oubliant que s'ils parlaient encore français, c'était à lui en grande partie qu'ils le devaient ${ }^{17}$. " Cette désapprobation lui perça le cœur. Il était tellement sensible. C'est à ce moment-là qu'il songea sérieusement à reprendre sa chère profession. A partir de ce jour aussi, dans chacun de ses discours, il est facile de percevoir que c'est un grand blessé qui parle. Le terme de solidarité apparaît comme un leitmotiv. Ce n'est pas pour rien... Sa résolution fut prise aussi : il donnerait sa démission comme député, advienne que pourra!

Il abandonna le poste à un plus jeune, M. Léodat Gauthier.

Le sénateur (1945-1955)

Les Franco-Ontariens avaient droit à un autre sénateur. La place de Louis Côté restait vide. Le 11 juin 1945, le gouvernement de Mackenzie King se décida enfin à nommer le docteur Hurtubise. C'était la deuxième fois que l'on choisissait un sénateur dans le Nord-Ontario et la première fois qu'un tel honneur rejaillissait sur Sudbury, notait monsieur Mason ${ }^{18}$.»

Comme il convenait, il y eut de grandes fêtes en son honneur, d'abord parmi les politiciens; puis chez les médecins, à l'hôpital SaintJoseph. Tous, Canadiens anglais et français s'unirent pour louer à qui mieux mieux les qualités du héros du jour... et le féliciter. Le maire Collins, de Copper Cliff, qui s'était joint au maire Beaton, de Sudbury, disait : Je l'ai connu en 1904... et il est toujours resté le parfait gentilhomme d'alors ${ }^{19}$.

17 Loc. cit.

18 Eminent Surgeon, dans The Sudbury Daily Star, 25 juillet 1945.

19 Loc. cit. 
Le juge T. Murray Mulligan, de la Baie du Tonnerre (Thunder Bay), résume tous ces éloges : " Je me demande où ils (les chefs libéraux) auraient pu trouver un meilleur homme ${ }^{20}$. »

Le témoignage de Mason est succulent : * Depuis trente ans que je connais le docteur Hurtubise et nous avons toujours fait bon ménage... Faut dire que c'était un travailleur et un parfait gentilhomme ${ }^{21}$. McCrea, un adversaire politique de la première heure, qui n'avait pu venir, lui envoyait tout de même un télégramme où il le félicitait de tout cœur.

Avant d'aborder avec délices le patriote gentilhomme, nous pourrions accumuler des faits prouvant sa parfaite intégrité. Qu'il suffise d'en rappeler un.

En 1930, une fois élu député, il y avait, dans ses livres de comptes, plus de $\$ 50,000$ d'arrérages, il aurait pu réclamer. Il prit, avant de se lancer définitivement dans la politique, une décision irrévocable qui montre sa générosité, une libéralité presque excessive. "Si je les conserve, je serai fort tenté à certains moments de regarder les noms de mes débiteurs avec dépit... Le mieux à faire, c'est de tout enterrer cela. " Il prit les fameux livres et les brûla. Puis, il sembla soulagé d'un grand poids ${ }^{22}$.

\section{LE PATRIOTE GENTILHOMME ${ }^{23}$}

Il l'emporte d'emblée sur le politicien. Le docteur prétend, comme son Maître le Christ, qu'il est venu d'abord pour "servir " les siens ${ }^{24}$. Comme le Christ, il est la modestie en personne. Ecoutez-le se présenter à la Société des Conférences d'Ottawa : "Je n'ai jamais eu la prétention d'être un littérateur ni un philosophe, encore moins un orateur ou un conférencier. Je ne suis qu'un pauvre chirurgien qui a fini par devenir un politicien. Fils de cultivateur, né dans le beau comté de Prescott, j'ai fait mes études primaires en Ontario, mes études classiques à Rigaud, et mes études médicales à l'Université Queen's (Kingston). C'est vous dire que je suis un mélange d'un peu de tout ${ }^{25}$.

Mais ce mélange était de qualité. Il lui fournira la science psychologique essentielle pour solutionner certains problèmes et une telle dextérité d'adaptation et de compréhension et de pacification que les obstacles s'évanouiront devant lui. Ces facultés réunies annoncent le chef. Ainsi cet heureux mélange, servi par une forte personnalité comme la sienne, lui permit de découvrir la clef du problème des atavismes chez les transplantés hors du Québec. Comme il en avait toutes les données, il a pu en mieux saisir la complexité des rouages et

20 Loc. cit.

21 Loc. cit.

22 Conversation avec Léodat Gauthier, ACSC, FH.

23 Ernest Comтe, Le sénateur Hurtubise, dans le Lien, VII, 6 mars 1955. Il cite Gustave Lacasse, de la Feuille d'Erable, février 1955.

24 Luc XXII, 27; Matth. XX, 8.

25 Raoul Hurtubise, Les Canadiens : 1. 
l'unique solution, la chrétienne. D'abord, il savait que nul n'a le droit de renier son passé ou ses ancêtres, même s'il devient majeur. Le jeune homme qui se marie n'a pas le droit de délaisser ses parents, encore moins de les mépriser. Pour l'homme marié, sa femme passe avant tout, c'est vrai. Le Christ et l'Eglise nous l'enseignent. Mais même pour celui qui prend femme, le quatrième commandement demeure : " Honore ton père et ta mère, afin de vivre longuement. » C'est une question de vie et de survie. Une génération n'est pas la fin d'un monde, elle en est le commencement! Le reste est révolutionnaire, contre nature, contre Dieu et les hommes. Puis, jamais le neuf ou l'avenir ne se bâtit solide, en dehors ou au mépris de l'histoire. Ainsi en est-il - toute proportion gardée - des peuples qui aspirent à l'indépendance légitime. Elle doit s'acquérir ou se conquérir, dans le respect des lois et la reconnaissance due à la mère patrie. Le docteur avait compris cela. Il demeura donc attaché à Québec, tout en se créant une patrie nouvelle en Ontario, qu'il n'a d'ailleurs jamais considéré comme une terre étrangère. Loin de là ! "Vous n'êtes pas des émigrés, ici. Vous êtes les descendants des premiers occupants ${ }^{26}$ ", rappellera-t-il énergiquement à ses compatriotes de Windsor, en leur parlant de fierté nationale.

\section{Le chef pacifique}

Depuis 1910 jusqu'à sa mort, son devoir patriotique lui apparut très clair : il était un chevalier au service de la cause des siens en terre ontarienne. Voilà tout! Et il ne fera plus qu'un avec le peuple canadien-français. L'âme d'un peuple passe avant le territoire. C'est pour cette âme française qu'il est décidé à vivre, à combattre et, s'il le faut, à mourir !

C'est dans les luttes scolaires qu'il commence vraiment à faire figure de chef pacifique. On était au tournant de l'épisode tragique du Règlement XVII. Organisateur, membre et président de la Commission scolaire ici, candidat malheureux, mais brave en 1923 : autant de phases d'une ascension vers une victoire décisive ${ }^{27}$. Il a lui-même décrit au long et au large cette épopée. Il a prouvé par des actes plus que par des mots que la cause de l'éducation lui tenait au cœur. En 1910, il assista au premier Congrès des Franco-Ontariens, à Ottawa. Il devint membre de l'exécutif de l'Association canadienne-française d'Ontario, position qu'il n'a cessé d'occuper qu'avec la mort. Dès 1912, il est membre de la Commission des Ecoles séparées de Sudbury. En 1915, il en devenait le président, poste qu'il conservera durant quinze ans. A son bureau, vrai chef-lieu des patriotes, l'on discutait des questions nationales et notamment de l'amélioration de la situation scolaire. Jamais il ne s'est agi néanmoins de lutter contre nos coreligionnaires ${ }^{28}$. Il obéissait en cela strictement aux directives des papes Pie X et Benoît XV. En 1923, pendant la lutte scolaire, il accepta la candidature libérale contre le

26 Discours du docteur Hurtubise à Windsor, le 27 octobre 1946, ACSC, FH.

27 Raoul Hurtubise, Ecoles bilingues de Sudbury, Sudbury, La Société historique du Nouvel Ontario, 1954, Coll. Documents historiques, $\mathrm{n}^{\circ} 28$ : 21-42.

28 Ibid. : 29. 
ministre des Mines, l'honorable Charles McCrea, il fut défait. Toutefois, sa campagne contre le Règlement XVII contribua, avec l'effort des siens dans les autres parties de la province, à l'abolition de cette loi inique, en 1927. Il est bon de le redire.

En 1912, il est un de ces pétitionnaires qui décidèrent les révérends Pères Jésuites à fonder un collège classique à Sudbury. Et le Collège Sacré-Cœur ouvrait ses portes dès septembre 1913. En 1915, avec l'abbé J.-A. Ménard, il fonde l'« Euvre des Etudiants». Il en resta le président depuis sa fondation jusqu'en 1944, lorsque l'on confiera l'œuvre à l'Association des Anciens. Cette œuvre avait pour but de créer un fonds pour venir en aide aux étudiants pauvres. Il se souvenait d'avoir été de ceux-là !

Lors des fêtes du quatrième centenaire de la Compagnie de Jésus, le docteur évoqua les sacrifices des missionnaires Jésuites, et après avoir énuméré toutes les paroisses qu'ils avaient fondées, il terminait en faisant l'éloge du Collège classique : "Mais l'œuvre par excellence que la Compagnie de Jésus a établie dans ce diocèse, c'est le Collège SacréCoeur ${ }^{29}$. »

Son frère Henri, alors clerc de Saint-Viateur, admirant son zèle pour le Collège Sacré-Cœur, le taquinait un brin et finissait son épître par ces mots qu'il voulait badins et qui sont restés profondément vrais : "Saluts au docteur Ignace ${ }^{30 ! » ~ A m i ~ d u ~ C o l l e ̀ g e, ~ i l ~ e ́ t a i t ~ l e ~ m e ́ d e c i n ~}$ officiel des élèves et de la communauté.

En 1945, il a tenu plusieurs assemblées privées et fait de nombreuses démarches pour obtenir le titre d'Université au Collège Sacré-Cœur. Il fit de même pour stimuler la campagne de souscription en faveur de sa seconde Alma Mater. Dans un de ses discours, il souligne la valeur de l'enseignement des classiques : "Une nation vaut ce que vaut son élite intellectuelle. Nous, Canadiens français, nous pouvons être fiers de notre passé dans le domaine de l'éducation. Nous sommes fidèles aux traditions de l'ensignement classique catholique. »

Le 23 novembre 1950, les Anciens de Bourget l'élisaient président général de leur Association.

En 1951, il léguait au Collège Sacré-Cour quelques-unes de ses bibliothèques et, à sa mort, plus de 300 volumes. Il est un de nos bienfaiteurs insignes.

Pour promouvoir la fierté des siens, il avait résumé le Bref historique des Canadiens français de l'Ontario, où l'auteur (le R.P. Arthur Joyal, o.m.i.) prouve que de l'est à l'ouest, du sud au nord, l'Ontario a été exploré, habité d'abord par de grands Français. Il l'avait toujours à la mémoire et le servait au bon moment pour raffermir les volontés chancelantes ou pessimistes.

29 Emilien Chiasson, Le docteur J.-Raoul Hurtubise parle des activités des Jésuites dans la région de Sudbury, dans le Droit, 22 février 1941.

30 Lettre de H. Hurtubise à son frère, 10 mai 1916, ACSC, FH. 
L'éloge de la ténacité ancestrale, qu'adressait à notre groupe ethnique en général le publiciste Chauveau, semble s'appliquer mot à mot à la pléiade de chefs de 1910. Or le docteur Hurtubise était, dès 1910, l'un des chefs reconnus du groupe français du Nouvel-Ontario et il était à la célèbre convention qui a vu naître l'organisme sauveur de l'A.C.F.E.O., dont il a été membre actif et longtemps l'âme dirigeante du Comité régional.

Si le vent de l'anglicisation et de la protestantisation - nos deux

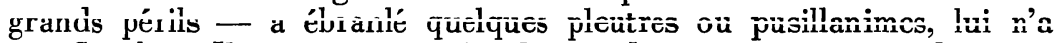
pas flanché. Il avait, comme la plupart de ses compatriotes dispersés hors de la province mère, emporté avec lui « cette puissance énergique et insaisissable " qui "comme le génie, notait Chauveau, échappe à l'astuce de la politique ». Non, il n'a pas courbé sous ces rafales, mais il a maintenu et accru l'héritage laurentien en Ontario. Il n'y avait pas de pire crime pour lui que de trahir cet héritage sacré. Par ses exemples et par ses discours, il a protesté contre les défaitistes et ceux qui nous traitent en intrus ou émigrés en cette province. Patriote, il l'était par conviction : s'il défendait vigoureusement des principes, des droits, il détestait à mort la manie des chicanes.

Il est allé plus loin. On peut dire qu'il s'est constitué l'apôtre de l'avancement des nôtres. "La défense des intérêts culturels, religieux et scolaires » a été « l'une des principales préoccupations de sa vie ${ }^{31}$."

A cette fin, il fit partie, comme membre actif et bienfaiteur, de nombreuses sociétés qui favorisaient cet avancement. Nommons l'Institut canadien de Sudbury, le Club Saint-Louis, la Société historique du Nouvel-Ontario, la Société canadienne d'Histoire de l'Eglise, les Richelieu, la Radiodiffusion bilingue, puis française, à Sudbury, etc.

Il ne ménage pas les vifs encouragements aux plus jeunes qui veulent hausser le niveau intellectuel paroissial en fondant un journal, etc.

La Société historique locale n'oubliera jamais ses fructueuses démarches consenties pour lui procurer des volumes et sa ferveur déployée à stimuler les nôtres au travail historique et à la cueillette des documents.

L'histoire régionale le passionnait et, à travers ses notes, s'empilent des ébauches de généalogies des pionniers de Sudbury. Comme toujours, il prêchait d'exemple. Il composa d'abord La vie du docteur Howey ${ }^{32}$, puis une longue étude sur Les Ecoles bilingues de Sudbury, un tract sur Les Canadiens français et le Nouvel-Ontario. Partout, il fustige la paresse de nos professionnels et prône la culture plus poussée des nôtres, en général. Il y reviendra inlassablement dans ses discours, soit à Rigaud, soit à Sudbury. Il recommandait aussi la solidarité ou coordination de nos forces : autant de mots d'ordre d'un chef! Relisez

\section{L'Heureux, ibid.}

32 Raoul HurtuBise, Le docteur William H. Howey, Les familles pionnières, Sudbury, La Société historique du Nouvel-Ontario, 1944, Coll. Documents historiques, $\mathrm{n}^{\circ} 5: 33$. 
ses consignes : « Il n'y a pas d'hommes parmi les moins de quarante ans qui n'aient bénéficié de ses activités. Il a été un Canadien idéal, en ce sens qu'il n'a pas recherché seulement les avantages du Canada français, mais du Canada tout entier... » Ces mots élogieux de Son Honneur le juge J.-S.-A. Plouffe traduisent les sentiments unanimes, sans farder la vérité ${ }^{33}$.

Le peuple reconnaît vite ses chefs dans les natures modestes, aimables, judicieuses, dévouées : il a l'intuition juste des valeurs. En haut lieu aussi, on a reconnu dans le docteur Hurtubise l'homme d'élite. Il a recueilli des récompenses, des témoignages non équivoques. Tous s'accordaient à souligner les mérites signalés qu'il a prodigués aux siens. A Sudbury, la Société historique était fière de le choisir comme premier président; la Société canadienne d'Histoire de l'Eglise répétait le même geste et s'honorait comme notre Société de le compter longtemps, par la suite, parmi ses directeurs ou officiers. Enfin, en 1952, l'Université Laval lui décernait un doctorat ès Lettres pour services insignes rendus à la langue française.

\section{Les derniers temps (1952-1955)}

Le purgatoire de la douleur est le chemin montant des élus. Tous ont passé par ce creuset. Seul le chemin de la croix mène à la béatitude. Retiré à l'hôpital Saint-Vincent d'Ottawa, après deux ans et demi de pénibles souffrances, parfaitement résigné et muni des sacrements de sa mère la sainte Eglise, le docteur Hurtubise, âgé de soixante et onze ans et sept mois, rendait sa belle âme à Dieu, le 31 janvier 1955.

Sa mort a été le digne parachèvement d'une vie pleine de suavité, d'humilité et de charité. Et quelle vie continuellement droite et montante! Tissée comme un antique gobelin avec les menus fils des quotidiennes fidélités aux grandes causes, elle demeurera une œuvre d'art discrète, mais immortelle - comme il les aimait tant, en songeant sans doute à son père et à sa mère - un pur chef-d'œuvre élaboré uniquement à la plus grande gloire de Dieu !

$\mathrm{Ne}$ convenait-il pas de faire revivre - ne fut-ce que peu de temps - le chirurgien consciencieux et insigne, le catholique exemplaire, le politique d'un genre à part, le patriote gentilhomme et le noble sénateur, en ces assises de votre auguste Société tenues à Sudbury? Ne s'était-il pas délibérément identifié avec sa ville adoptive au point de ne faire plus qu'un avec elle? Lui, le passionné d'histoire régionale, le méritait peut-être plus que tout autre. Je n'ai fait en tout cas que répondre à l'un de ses plus ardents souhaits. N'est-ce pas lui qui voulait qu'à la Société historique l'on fit surgir les "grands modestes", afin que les jeunes générations sachent honorer "les humbles héros qui ont bien mérité de la patrie ». Comme il avait raison, c'est « un pieux devoir » plus opportun que jamais de rappeler à des jeunes trop enclins à oublier un riche passé que "la petite patrie de ces régions du nord ontarien " a été féconde " en hommes de modeste extraction qui sont devenus 
grands, grâce à un courage, un esprit d'entreprise, une énergie, une détermination et une vaillance dignes de toutes les admirations ${ }^{34}$."

Bien plus, pour nous, ex-recteur du Collège Sacré-Cœur, sudburien d'adoption et membre fondateur de la Société historique du NouvelOntario comme lui, le faire revivre quelques instants devant vous, c'était tout bonnement et avant tout acquitter officiellement une immense dette de justice et de gratitude.

Les nations fières, reconnaissantes, gravent dans le bronze pour les immortaliser les noms de leurs fils glorieux qui ont passé, diffusant le parfum du Christ, en faisant le bien! Quand donc lirons-nous, audessus de la porte de la plus belle salle d'opération de l'hôpital SaintJoseph, le nom du docteur Joseph-Raoul Hurtubise en lettres d'or? Quand donc les visiteurs rencontreront-ils à l'endroit le plus beau et le plus en vue de Sudbury un monument somptueux qui soit un peu à la hauteur de son inépuisable dévouement et de l'élévation de sa vie? Qu'attendent pour poser un si noble geste ses innombrables débiteurs canadiens-français, anglais ou néo-canadiens? Qu'ils aient tous assez le sens de la fraternité chrétienne pour s'unir et prouver leur magnanimité en sortant de l'ombre celui qui a et le mieux et toujours dépensé ses énergies à glorifier sa ville?

Le moment a sonné d'aller au-delà des discours et des prières. Passons aux actes! Grâce à nous, il faut que les générations montantes se souviennent et se grandissent jusqu'à l'immortalité à leur tour, en admirant et imitant les vertus incarnées dans ce grand chrétien qui fut si fièrement et si dignement leur aîné !

\author{
Guy Courteau, s.j., D.Ph. \\ Professeur d'Histoire à l'Université de Sudbury.
}

34 Raoul Hurtubise, Le docteur William Howey, Documents historiques, $\mathrm{n}^{\circ} 5$. 